\title{
What Role does the Voice Behavior Play in the Relationship between Mianzi and Innovative Behavior?
}

\author{
Ping WANG ${ }^{a}$, Shao-Qi WANG ${ }^{\text {,** }}$
}

School of Economics and Management, Zhejiang Sci-Tech University, Hang Zhou, China, 310018

awp190923@163.com, b15858133483@163.com

Keywords: Mianzi, Voice behavior, Innovative behavior.

\begin{abstract}
The behavior of Chinese people is influenced by the concept of Mianzi. When individuals want to save Mianzi, they will be glad to express himself more actively so as to show more voice behavior and innovative behavior. On the contrary, be afraid of losing Mianzi will prohibit their voice and innovative behaviour. And the saving Mianzi can affect positive predictive voice behaviour so as to promote innovative behaviour, while the afraid of losing the Mianzi is the opposite.
\end{abstract}

\section{Introduction}

With the transformation of made in China into created in China, innovation has been repeatedly mentioned. Chinese companies need to have enough energy and innovation to stay competitive in a rapidly changing market, and enterprise innovation is based on individual employee's innovation. Innovation is the realization of economic development through invention and discovery, it is not just as a technical concept but also an economic concept (Schumpeter, 1934). So for enterprise staff, individual innovation is not only for technical positions.

Innovative behavior is a kind of behavior which is out of the role, not only affected by enterprise's incentives, but also by the factors such as culture. In the Chinese cultural background, individual employees generally have conservative ideas and focus on Mianzi. The behavior of Chinese people is influenced by the concept of Mianzi. Innovative behaviors are risky and challenging, and employees may not innovate in order not to lose Mianzi(Begley, Tan, 2001; Leung, Chen, Zhou, 2014). At the same time, in an uncertain situation, an individual who has strong Mianzi concept may be afraid of the adverse effect of his suggestions which may cause his own and others' Mianzi damaged so as not to take advice. (Ashford, Northcraft, 1992). Voice behavior is an act of expressing one's own innovative proposals and suggestions for improvement (LePine, Van Dyne, 1998), which itself is an individual innovative behavior. So, Mianzi will affect voice behavior and at the same time affects the creation of innovative behavior. Therefore, it is necessary to study the relationship among Mianzi, voice behavior and innovation behavior.

\section{Theoretical Basis}

\section{What is Mianzi?}

Mianzi is the recognition of an individual in his social status (Xian-Jin HU, 1944), social contribution (Goffinan, 1967; Yao-Ji JIN, 1988)and the respect from others due to his position and accomplishment (Ho, 1976), it can be seen as a function of social status or prestige(Guang-Guo HUANG, 1986). Mianzi can also be seen as a self-psychological image, is generated for the universal norms in society and other people's perception of individual evaluation (Zhi-Zhao CHEN, 1988;Xue-Wei ZHAI, 2011), is the positive image individuals want to maintain in a certain relationship(Lim, 1994; Ting-Toomey, 1998).

There are many ways to divide the dimensions of Mianzi. Such as self-Mianzi and other-Mianzi(Goffmam, 1955);positive Mianzi and negative Miazni (Brown, Levinson, 1987); self-Mianzi, other-Mianzi and other Mianzi concern(Ting-Toomey, 1988);autonomy Mianzi, fellowship of Mianzi and competence of Mianzi(Lim, 1994);acquisitive Mianzi and protective 
Mianzi(Mei-Ling ZHOU, 1997);Mianzi of ability, relational Mianzi and moral Mianzi (Gong-Min BAO, Zhuo-Jia ZHAO, 2009);save Mianzi and afraid of losing Mianzi(Xin-An ZHANG, 2012).

At present, combing with Chinese background, domestic scholars have created a lot of measurement scale. Such as the acquisitive and protective Mianzi-oriented measurement scale(MeiLling ZHOU, 1997), the scale was used to study the relationship between the concept of Mianzi and shopping behavior (Zheng-Lin ZHANG, Gui-Jun ZHUANG, 2008); and the Need of Mianzi scale (including two sub-scales of loving Mianzi and thin Mianzi) and a virtual Mianzi-like sub-scale (Zhi-Zhao CHEN, 2006);Xin-An ZHANG(2012)based on previous studies and combined Chinese background, developed the Mianzi perspective scale contains saving Mianz and afraid of losing Mianzi two dimensions of 11 questions.

\section{What is Voice Behavior?}

Voicebehavior is a change-oriented approach to improving the environmentand is a constructive interpersonal behavior(Borman, Motowido, 1997;Jin-YunDUAN, Jia-NanZHONG, 2005). Its purpose is to provide ideas or perspectives and to generate positivedaring challenges and constructive improvements in order to accomplish the task of a team or organization and to improve organizational performance(De Dreu et al. , 2001;Detert , Burris, 2007;Liu, Zhu, Yang, 2010).

Many studies show that the act of voice behavior is not a single dimension but a multidimensional and complex concept (Withey, Cooper, 1989). Divided by motivation as Conscious voice behavior, unconscious voice behavior, and conscious silent behavior(Hischman, 1970);or divided into pro social voice behavior, defensive voice behavior, and acquiescence of behavior(Van Dyne, Ang, Botero, 2003). There are also some scholars divide the object of voice into two groups: executive director and direct chief executive (Ioannis et al. , 2008); or voice to colleagues and voice to superiors (Liu et al. , 2010). Liang \& Farh (2008) rely on the previous research and Chinese background, the behavior is divided into promotive voice and prohibitive voice. According to the doctrine of the mean of Chinese traditional culture, another scholar divided the voice behavior into self-aggressive voice and considerate the overall situation of voice(Jin-Yun DUAN, Bin LING, 2011).

Due to the diversity of the dimensions of voice behavior, the measurement methods are also different. Such as the Hagerdoorn et al. (1999) development of the caring voice and aggressive voice two-factor scale. On the basis of foreign studies and combined with the background environmental factors in China, Liang\&Farh(2012) developed a two-dimensional voice behavioral questionnaire for both promotive and prohibitive. At present, this scale is the most widely used measurement scale by domestic scholars(Rui LI , Wen-Quan LING, Shi-Shun LIU, 2009;Rui LI, Wen-Quan LING, Li-Luo FANG, 2010;Chang-Chun XIANG, Li-Rong LONG, 2013; Jian LIANG, 2014).

\section{What is Innovative Behavior?}

From the individualistic perspective, innovation is a capability and willingness. For example, Kirton (1976) argued that innovative behavior is a way to break the routine and take a fresh approach to solve problem. Hurt etal. (1977)argued that personal innovation should be defined as the willingness to change. However, most scholars believe that innovation is a process. Vandeven(1986) argued that innovation was based on the practice of developing, implementing, reacting and amending the realization of innovative ideas. The essence of innovation lies in the innovation of ideas. Personal innovation is a complex process, which can be divided into three stages: the first stage is the individual contact problem and have a cognition, the second stage is the innovation individual seeks supporters, the third stage is the individual visualizes their creativity to get the prototype of innovation (Kanter, 1988). In addition, innovation behavior can be regarded as the process of generating, implementing and completing the three phases (Scott\&Bruce, 1994). Many Chinese scholars also agree with this theory (Xiao-Jun LU, Guo-Liang ZHANG, 2007; Yuan-Dong GU, Ji-Sheng PENG, 2010).

At present, the most commonly used dimension of innovative behavior and measurement scale is the single-dimension scale which is developed by Scott \& Bruce (1994). The scale also has a high 
reliability and validity in the study (Jing-Ji WU, He-Mao LIN, 1998; Ting-Ting ZHANG, 2009; Yun LIU, Jin-Tao SHI, 2009). Based on this scale, Zhou\&George(2000)later adapted a 13-item single-dimension scale, which was mainly measured by managers' evaluation of employee's individual innovative behavior.

\section{The Relationship between Mianzi and Innovation Behavior}

In asocial environment like China which is concerned about the Mianzi, it is indispensable to get Mianzi and save Mianzi(Rui-LingZHU, 1988). When faced with a specific situation that requires decision-making, the perception of pros and cons of Mianzi is an important factor which affect the individual's behavior(Zhang, Ting-Toomey, Oetzel, 2015).

In the process of innovative decision-making, the individual's innovative behavior can be restrained if the individual anticipates that his new idea will not be accepted or will be opposed by others, and his Mianzi is lost. (Begley, Tan, 2001; Leung, Chen, Zhou, 2014). Chinese attach importance to interpersonal relationships. And Mianzi has the characteristics of mutual exchange(Ho, 1994). Individuals will also choose to retreat without innovating if their own thinking can make others have no Mianzi in the course of innovative behavior(Zhuo-Jia ZHAO, 2013;Leung, Chen, Zhou, 2014). On the contrary, employees tend to save Mianzi to promote their innovative behavior (Begley, Tan, 2001). If in the process of innovation, we can predict that it is helpful to save Mianzi by challenging the authority, putting forward new ideas, breaking the traditional thinking, the individual will show positive behavior, and try to make more innovations (Min ZHANG, 2013).

From these, we can see that the Mianzi-saving has a positive predictive effect on innovative behavior, be afraid of losing Mianzi has a negative predictive effect on innovative behavior.

\section{The Relationship between Mianzi and VoiceBehavior}

In the Chinese cultural background, Mianzi is an indispensable factor. For Chinese employees in the enterprise, everyone has a Mianzi-saving psychology, which affects their thinking and behavior in the work. From the perspective of saving or losing Mianzi, the researcher finds that trying to save Mianzi can make the individual show more obvious voice behavior, while the opposite psychology of losing Mianzi can inhibit individual voice behavior.

Individual longing for saving Mianzi can lead to some individual initiative behaviors. A positive external image can help employees to obtain satisfying social outcomes such as recognition, friendship and authorities, as well as some other people's resources (Leary, Kowalski, 1990; Ashford, Tsui, 1991). In the organization, employees can make suggestions to let the leaders or colleagues know themselves, and get the positive result through being appreciated by others, so as to achieve the purpose of saving Mianzi. Yi-Nan WANG and Zhong-Fang YANG (2007) pointed out that those who have strong desire to save Mianzi will correct the organizational rules and make innovative suggestions, and employees will tend to voice.

Compared with the influences of saving Mianzi, the loss of Mianzi has more serious consequences, people may not have to try to save Mianzi, but the loss of Mianzi may lead to inability to establish himself in the society(Ho, 1976). Employees may be worried about their own image being affected, so as not to put forward their own ideas. Ashford, Rothbard, Piderit, and Dutton (1998) argue that if the voice behavior of the proposed person is not successful, other employees may think that the speaker is harmful or incompetent to the organization, which will damage his or her image. Therefore, they think individuals who are more sensitive to the possibility of losing their image, the less likely they will tend to voice. Fear and worry are the factors that cause employees to behave in a silent manner. Employees fear that they will leave a negative image in others' minds and disrupt their relationships so as to choose silence (Morrison, Milliken, Hewlin, 2003; Qiu-Hong YANG, 2016). It can be speculated that saving Mianzi have the positive predictive on voice behavior, and be afraid of Mianzi have the negative predictive effect on voice behavior. 


\section{The Relationship between VoiceBehavior and Innovative Behavior}

Innovative behavior itself is a challenge to the existing work processes and presents new and valid ideas when problems are discovered (LePine, Van Dyne, 1998). Voice behavior can be divided into promotive voice behavior and repressive voice behavior. Promotive voice behaviour is that employees express their views and new ideas in order to improve the performance of organizations or teams (Liang, Farch, 2012). In the course of innovation behavior, employees will firstly put forward their ideas and then exchange ideas with colleagues or leaders. Combined with promotive voice behaviour, it is not difficult to find that promotive voice behaviour itself is a individual behaviour which has innovative tendency(Li-Li LIU, 2017).

Many studies show that voice behavior can enhance employees' innovative awareness and ability, thus promoting their innovative behavior. De Dreu \& Van Vianen (2001)argued that the individual voice behavior of employees can stimulate innovative behavior. Employees can introduce their ideas to organizational members or seek new ways to solve problems in order to achieve better performance. Voice behavior is an organizational behavior that activates innovative ability, and has a direct relationship with the emergence of innovative behaviour (Dyne, Ang, Botero, 2003). Employees' knowledge is important for innovation (Amabile, 1994), Lynch (2007) emphasizes the importance of employees' knowledge to organizational innovation. He believes that organizations need to have the ability to utilize employees' knowledge, which is closely linked with voice behavior. Employees' voice behavior level will have a significant impact on team's innovation performance, which will affect employee's individual innovation behavior(Gong-Min BAO, Yuan-Yuan QIAN, 2009). Ng\&Feldman (2012) found that the voice behavior not only has a positive effect on the performance of employees, but also has a strong predictive effect on employee's individual innovative ability and implementation of innovative ideas.

As a result, it can be speculated that the voice behavior has a positive predictive effect on innovative behavior.

\section{Theoretical Model}

As an individual initiative behaviour, voice behaviour is affected by individual and external factors. In the context of Chinese culture, Mianzi is a very important factor in the work process which affects the behaviors of employees. An individual who wants to save Mianzi can achieve this purpose by expressing himself/herself actively to get other's recognition and appreciation of others. They adopt voice behaviour to achieve their goals in the work(Qiu-Hong YANG, 2016). Conversely, if an individual is afraid of losing Mianzi, he will appear silent instead of making voice behaviour, so as to protect himself from losing Mianzi(Ashford, Rothbard, Piderit, Dutton, 1998). Therefore, Mianzi has an impact on voice behaviour, regardless of whether the individual wants to promote voice behaviour to save Mianzi or be afraid of losing Mianzi by suppressing voice behaviour.

The emergence of the voice behavior has greatly promoted the staff to express their thoughts and innovative ideas, and in the process there was innovative behavior. Because innovation itself is a challenge to the existing work processes, some new and valid ideas are presented when problems are discovered (LePine, Van Dyne, 1998). Therefore, there are some similarities between the innovative thinking and innovative behaviour in the process of making suggestions. Voice behaviour can greatly promote individuals to boldly put forward their innovative ideas in the innovation process so as to get the support of organization and then implement their ideas and finally achieve innovative results. Therefore, it can be presumed that the voice behavior plays an intermediary role between Mianzi and innovative behavior.

According to the above analysis, we can get the theoretical model, shown in Figure 1: 


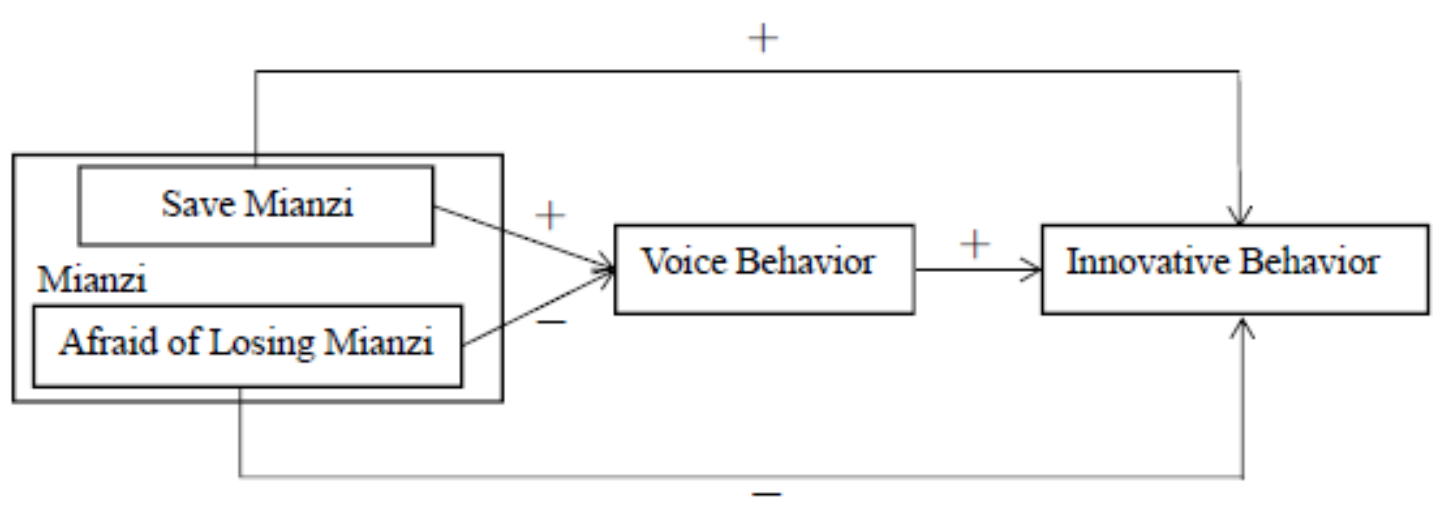

Figure 1 Theoretical Model

\section{Summary}

Mianzi has different effects on different dimensions of employees' voice behavior and innovative behavior, save Mianzi dimension plays a role in promoting employees' voice behavior and innovation behavior; on the contrary, afraid of losing Mianzi dimension can have an inhibitory effect on both of them. The voice behavior as an intermediary role in the relationship between the Mianzi and the innovative behavior, and the Mianzi has an effect on individual innovative behaviour by influencing the appearance of the invoice behaviour.

Therefore, enterprises should attach great importance to the role of Mianzi towards voice behaviour and innovative behaviour in the process of management. In the cultural background of China, Chinese cares a lot about the opinions of others and social evaluations. Chinese employees also think highly of leaders' ideas and colleagues' evaluation. In order to save Mianzi, employees will have more voice behaviors and innovative behaviors. On the contrary, afraid of losing Mianzi will make employees not to make suggestions and innovations, as suggestions and innovations may change the status quo, or harm the interests of others. In order not to conflict with others, they will choose to maintain the status quo instead of making voice behaviour and innovative behaviour.

Thus, enterprises should maintain the Mianzi of Chinese employees. For example, appropriate or not should be a judgement basis of staffs' suggestions rather than good or bad. And no matter whether the staff's advice and innovative ideas will help, they should be encouraged and supported by the enterprise. In addition, managers should also pay attention to the concept of employee's Mianzi in their management, they can use the Mianzi's concept of different employees through the way of position transfer. Employees who wants to have Mianzi can be transferred to position with higher innovative requirements, and the employees who are afraid of losing Mianzi should be placed on a position like administration.

\section{References}

[1] Begley TM, Tan WL. The Socio-Cultural Environment for Entrepreneurship: A Comparison Between East Asian and Anglo-Saxon Countries, J. Journal of International Business Studies, 2001, 32(3):537-553.

[2] K Leung, Z Chen, F Zhou, L KaiT. he role of relational orientation as measured by Mianzi and renqing in innovative behavior in China: An indigenous analysis, J. Asia Pacific Journal of Management, 2014 , 31 (1) :105-126.

[3] SJ Ashford, GB Northcraft. Conveying More(or Less)Than We Realize: The Role of Impression-Management in Feedback-Seeking, J. Organizational Behavior \& Human Decision Processes, 1992 , 53 (3) :310-334.

[4] LePine J A, Van Dyne L. Predicting voice behavior in work groups, J. Journal of Applied Psychology, 1998, 83(6): 853-868. 
[5] Ho. DYF. On the concept of Mianzi, J. The American Journal of Sociology, 1976(81): 867-884.

[6] Ting-Toomey S. Mianzi work competence in intercultural conflict: An update Mianzi-negotiation theory, J. International Journal of Intercultural Relations, 1998(22), 187-225.

[7] Xin-An ZHANG. Chinese people's Mianzi and show off rich luxury spending behavior, J. Journal of Marketing Science, 2012, 23 (6): 75-93. In Chinese.

[8] Borman, W. C., Motowidlo, S. J. . Task performance and contextual performance: The meaning for personnel selection research, J. Human performance, 1997, 10(2), 99-109.

[9] Jin-Yun DUAN, Jian ZHONG. An organization's remorse behavior, J. Psychological Science, 2005, 28 (1), 69-71. In Chinese.

[10]Detert, J. R., Burris E. R. Leadership behavior and employee voice: Is the door really open?, J. Academy of Management Journal, 2007, 50(4), 869-884.

[11]Liu W, Zhu R,Yang Y. I warn you because I like you: Voice behavior, employee identifications, and transformational leadership, J. Leadership Quarterly, 2010, 21(1), 189-202.

[12]Ioannis Nikolaou, Maria Vakola, Dimitris Bourantas. Who speaks up at work? Dispositional influences on employees' voice behavior, J. Personnel Review, 2008, 37(6), 666-679.

[13]Liu W, Zhu R, Yang Y. I warn you because I like you: Voice behavior, employee identifications, and transformational leadership, J. Leadership Quarterly, 2010, 21(1), 189-202.

[14]Jian LIANG. Ethical leadership and employee advice: a regulatory - the construction and testing intermediary model, J. Acta Psychologica Sinica, 2014, 46 (1), 1-13.

[15]Hurt, H. T., Joseph K\&Cook C. D.. Scales for the measurement of innovativeness, J. Human Communication Research, 1977, 4(1):58-65.

[16]Vandeven, A. H. Central problems in the management of innovation, J. Management Science, 1986, 32: 590-607.

[17]Scott, S. G., Bruce, R. A.. Determinants of innovative behavior: A path model of individual innovation in the workplace, J. Journal of Academic management, 1994, 37 (3) :580-607.

[18]K Leung, Z Chen, F Zhou, L KaiT. he role of relational orientation as measured by Mianzi and renqing in innovative behavior in China: An indigenous analysis, J. Asia Pacific Journal of Management, 2014 , 31 (1):105-126.

[19]Zhuo-Jia ZHAO. The Internal Mechanism of Mianzi-to-Mianzi Job Selection Conflicts among Knowledge Workers-An Empirical Study Based on Expectation Theory, J. Finance and Economics, 2013, (4): 101-106. In Chinese.

[20] Li-Li LIU. Research on the Relationship between Employee's Voice Behavior and Innovative Performance-With Organizational Support as a Regulating Variable, D. Capital University of Economics and Business, 2017. In Chinese.

[21]NgT WH, Feldman D C. Employee voice behavior: A meta-analytic test of the conservation of resources framework, J. Journal of Organizational Behavior, 2012, 33(2): 216-234. 